\title{
Use of culture and immunochromatographic technique for diagnosis of trichomoniasis in Sri Lanka
}

\author{
H Banneheke ${ }^{1}$, R Fernandopulle ${ }^{2}$, S Prathapan ${ }^{3}$, G de Silva ${ }^{1}$, N Fernando $^{4}$, R Wickremasinghe $^{1}$ \\ (Index words: trichomoniasis, Sri Lanka, laboratory diagnosis)
}

\begin{abstract}
Summary
As a majority of the trichomoniasis patients are asymptomatic, laboratory tests are crucial in case detection. The usefulness of culture and immunochromatographic technique (ICT) compared to microscopy for detection of trichomoniasis in Sri Lanka was assessed. Females (16-45 years) from Colombo district were screened for Trichomonas vaginalis using three laboratory tests namely, microscopy of wet smear, Trichomonas liquid culture and ICT (OSOM ${ }^{\circledR}$ trichomonas rapid test). Trichomoniasis by at least one test being positive was $4.8 \%$. Microscopy, culture and ICT detected $2.8 \%, 4.2 \%$ and $10 \%$ cases respectively. Microscopy missed $32 \%$ of culture positives. ICT is a simple, practical and reliable alternative to microscopy in laboratory diagnosis of trichomoniasis.
\end{abstract}

Ceylon Medical Journal 2013; 58: 122-123

\section{Introduction}

Trichomoniasis caused by Trichomonas vaginal is is a sexually transmitted infection, accounting for an annual incidence of 174 million cases with 44\% (76.5 million) occurring in South and South East Asia [1]. The prevalence of T.vaginalis in clinic based populations in Sri Lanka has varied from $4.4 \%-7.2 \%[2,3]$. The prevalence of asymptomatic infection may vary from $50 \%-85 \%$ in females, thus screening is vital for case detection [4]. Wet mount microscopy with sensitivity of $52 \%-72 \%$ is used commonly in routine practice worldwide $[5,6]$. Other investigations include microscopy of stained preparations, culture, polymerase chain reaction (PCR) and immunochromatographic technique (ICT) for Trichomonas antigens.

No studies have been done on the use of ICT for the diagnosis of trichomoniasis in Sri Lanka. The objective of this study was to evaluate the usefulness of culture and ICT compared to wet mount in the Sri Lankan setting.

\section{Methods}

A total of 601 females aged 16-45 years from the Colombo district were screened for T.vaginalis, irrespective of the presence or absence of symptoms. Study participants were recruited over a period of 18 months in 2007-2009. Those who refused vaginal examination, were on antibiotics or having any form of vaginal bleeding were excluded. Using speculum, three vaginal swabs from the posterior fornix were obtained. Trichomonas culture "Trichomonas modified CPLM medium base" ("HIMEDIA ${ }^{\circledR}$ " Ref M 460) was inoculated at the bed side and incubated at $37^{\circ} \mathrm{C}$. They were examined microscopically daily till the 5 th day for motile trichmonads. ICT OSOM ${ }^{\circledR}$ trichomonas rapid test (genzyme Diagnostics, Kent, ME194AF) was performed according the manufacturer's instructions for only 100 (17\%) participants due to financial constraints. Microscopic examination of the wet smear was done at the bed side under $\times 10$ and $\times 40$ objectives for motile trichmonads. The study was approved by the Ethical Review Committee of Faculty of Medical Sciences, University of Sri Jayewardenepura.

\section{Results}

The mean age of the participants was 31.7 years. The confirmed cases of trichomoniasis by at least one positive laboratory test were $4.8 \%$ (29/601) with 2.8\% (17/601) by wet mount microscopy, $4.2 \%$ (25/601) by culture and 10\% (10/100) by ICT. Wet mount was unable to detect 32\% (8/ 25) of culture positive infections (Table 1 ). Out of the 10 ICT positives, four were culture negative (Table 2 ). The sensitivity and the specificity of vaginal smear were $68 \%$ and $100 \%$ and the positive and negative predictive values were $100 \%$ and $98 \%$ respectively. The ICT had 100\% sensitivity, $96 \%$ specificity, $60 \%$ positive and $100 \%$ negative predictive values. In all these instances culture was taken as the gold standard.

Departments of ${ }^{1}$ Parasitology, ${ }^{2}$ Gynaecology and Obstetrics, ${ }^{3}$ Community Medicine and ${ }^{4}$ Microbiology, Faculty of Medical Sciences, University of Sri Jayewardenepura, Sri Lanka.

Correspondence: HB, email: <hasini.banneheke@gmail.com>. Received 13 September 2012 and revised version accepted 13 March 2013. Competing interests: none declared. 
Table 1. Comparison of vaginal smear with liquid culture of participants

\begin{tabular}{lccc}
\hline & $\begin{array}{c}\text { Culture positives } \\
(n=25)\end{array}$ & $\begin{array}{c}\text { Culture negative } \\
(n=576)\end{array}$ & $\begin{array}{c}\text { Total } \\
(n=601)\end{array}$ \\
\hline Wet smear & & & \\
Positive & 17 & 0 & 17 \\
Negative & 8 & 576 & 584 \\
\hline
\end{tabular}

Table 2. Comparison of immunochromatographic technique (ICT) with liquid culture of participants

\begin{tabular}{lccc}
\hline & $\begin{array}{c}\text { Culture positives } \\
(n=6)\end{array}$ & $\begin{array}{c}\text { Culture negative } \\
(n=94)\end{array}$ & $\begin{array}{c}\text { Total } \\
(n=100)\end{array}$ \\
\hline ICT & & & \\
Positive & 6 & 4 & 10 \\
Negative & 0 & 90 & 90 \\
\hline
\end{tabular}

\section{Discussion}

ICT showed a higher detection rate microscopy. Infection would have been missed in 32\% if only microscopy was performed. The sensitivity and specificity of microscopy in our study is comparable with other studies [5-8]. The manufacturer states $83 \%$ sensitivity and 99\% specificity of ICT compared to 'culture and PCR combination' and 96\% sensitivity and 95\% specificity compared to wet mount. ICT showing a higher sensitivity than the culture in this study can be attributed to false positivity. ICT is based on the detection of T.vaginalis antigens rather than the live whole parasite, therefore such false positivity results are likely to occur [9]. ICT, having high levels of sensitivity and specificity, has been recommended by others as a good screening test especially for low prevalence settings like in Sri Lanka [9-10].

Microscopy has retained its place as the sole diagnostic test in Sri Lanka and many other countries, because it is easy to perform, inexpensive and available at the point of care. When compared with laborious culture techniques in microbiology laboratories, the trichomonas liquid culture is simple and easy to prepare, store and process in the laboratory. However, ICT seems an easy test which can be done at the point of care when compared with the less sensitive microscopy or culture that requires 72 to 120 hours to obtain a result. This study shows that wet mount should not be used as the sole diagnostic technique due to low sensitivity. Instead, a second test should be added to the routine microscopy. The decision regarding the selection of the second test can be taken by the individual laboratory considering all aspects. In conclusion, we recommend ICT as a simple, practical and reliable alternative to wet mount. We also recommend culturing to be established along with the microscopy at least in tertiary laboratories.

\section{Acknowledgments}

We would like to thank Mr. W. S. A. Silva and members of the Department of Parasitology, Faculty of Medical Sciences, University of Sri Jayewardenapura for their support. The study was funded by University of Sri Jayewardenapura (University grant ASP/06/RE/MED/ 2005/09).

\section{References}

1. Global prevalence and incidence of selected curable sexually transmitted infections overview and estimates. 2001; http:/ /whqlibdoc.who.int/hq/2001/WHO_HIV_AIDS_2001.02.pdf. Accessed 04/08/2012.

2. Perera J. Microbiological patterns in vaginitis. Ceylon Medical Journal 1994; 39: 91-4.

3. Herath S, Fernando D, Jayasinge S. Risk factors of Trichomonas vaginalis in women attending central Sexually Transmitted Diseases Clinic Sri Lanka. Retrovirology 2012; 9: 42.

4. Schwebke JR, Burgess D. Trichomoniasis. Clinical Microbiology Reviews 2004; 17: 794-803.

5. Wiese W, Patel SR, Patel SC, Ohl CA, Estrada CA. A metaanalysis of the Papanicolaou smear and wet mount for the diagnosis of vaginal trichomoniasis. American Journal of Medicine 2000; 108: 301-8.

6. Radonjic IV, Dzamic AM, Mitrovic SM, et al. Diagnosis of Trichomonas vaginalis infection: The sensitivities and specificities of microscopy, culture and PCR assay. European Journal of Obstetrics Gynecology and Reproductive Biology 2006; 126: 116-20.

7. Wendel KA, Erbelding EJ, Gaydos CA, Rompalo AM. Trichomonas vaginalis polymerase chain reaction compared with standard diagnostic and therapeutic protocols for detection and treatment of vaginal trichomoniasis. Clinical Infectious Diseases 2002; 35: 576-80.

8. Madico G QT, Rompalo A, McKee KT, Jr., Gaydos CA. Diagnosis of Trichomonas vaginalis infection by PCR using vaginal swab samples. Journal of Clinical Microbiology 1998; 36: 3205-10.

9. Zaki1 MM, Moussa HME, Hassanin OM. Evaluation of the OSOM Trichomonas Rapid Test for Detection of Trichomoniasis vaginalis. Parasitologist United Journal 2011; 4: 177-84.

10. Campbell L, Woods V, Lloyd T, Elsayed S, Church DL. Evaluation of the OSOM Trichomonas rapid test versus wet preparation examination for detection of Trichomonas vaginalis vaginitis in specimens from women with a low prevalence of infection. Journal of Clinical Microbiology 2008; 46: 3467-9. 